\title{
Um Sistema de Apoio à Decisão Baseado em Agentes para Tratamento de Ocorrências no Setor Elétrico*
}

\author{
Ronneesley M. Teles ${ }^{1}$, \\ Marcos Ivamoto ${ }^{1}$, \\ Leonardo H. S. Mello ${ }^{1}$, \\ Valdemar V. Graciano Neto ${ }^{1}$, \\ Cedric Luiz de Carvalho ${ }^{1}$
}

Resumo: Empresas fornecedoras de energia elétrica e mantenedoras das redes de distribuição lidam diariamente com a reparação de falhas na rede elétrica e alocação de veículos especializados para atendimento de ocorrências. Em geral, tais empresas dispõem de recursos humanos limitados para cumprir estas atividades. Este artigo apresenta um Sistema de Apoio à Decisão Inteligente (SADI) que propõe-se a apoiar estas tarefas oferecendo sugestões de como distribuir e alocar veículos para efetuar manutenções na rede elétrica. A inteligência do SADI é implementada com a tecnologia de agentes, uma abstração ideal considerando o caráter distribuído do problema em questão.

Keywords: Sistema de Apoio à Decisão, Multi-agentes, Companhias de Eletricidade.

\footnotetext{
${ }^{1}$ Instituto de Informática, Universidade Federal de Goiás (UFG)

Caixa Postal 131 - 74.001-970 - Goiânia - GO - Brasil Fone: +55 (62) 35211182 (FAX)

\{ronneesley | mivamoto | lhsmello | vgracianoneto | cedric.inf.ufg \}@gmail.com

* Versão preliminar deste artigo apresentada no WESAAC 2010 e escolhida como um dos 3 melhores artigos completos.
} 


\begin{abstract}
Electricity Companies daily deal with electrical distribution network reparation and specialist vehicles allocation to treat occurrences. In general, these companies have limited human resources to accomplish these activities. This paper presents an Intelligent Decision Support System (IDSS), which intends to support these tasks by offering suggestions about how to distribute and allocate vehicles to maintain distribution network. IDSS's intelligence is implemented with agent technology, an ideal abstraction if considered the distributed nature of the problem.
\end{abstract}

Keywords: Decision Support Systems, Multi-Agent, Electricity Companies.

\title{
1 Introdução
}

Companhias Distribuidoras de Energia Elétrica são empresas responsáveis pela distribuição da energia elétrica bem como pela manutenção de sua rede de distribuição na região de cobertura. A Companhia Energética de Goiás (CELG) é a responsável por estas atividades no Estado de Goiás.

Para administrar e reparar falhas, a região de cobertura é dividida em unidades gerenciais. Cada unidade gerencial é responsável pelo atendimento de reclamações referentes a falhas na rede elétrica em sua área. A unidade gerencial utilizada como cenário para este trabalho corresponde à Região Metropolitana de Goiânia.

A CELG realiza atividades comuns a outras companhias distribuidoras de eletricidade, quais sejam: atendimento e manutenção de falhas na rede elétrica e controle da frota de veículos para efetuar os atendimentos.

Para tomar providências em relação às falhas que ocorrem, companhias elétricas dispõem de veículos especializados denominados viaturas. Um atendimento consiste na associação de uma viatura a uma ocorrência. As viaturas são posicionadas de modo a otimizar o atendimento. Os despachantes são os humanos responsáveis por alocar as viaturas para atender as ocorrências registradas. As ocorrências são registradas pelo serviço de atendimento ao cliente e são encaminhadas para os despachantes.

O posicionamento de viaturas é realizado a cada início de turno. O dia é dividido em três turnos de oito horas. A modelagem da distribuição é feita manualmente e com base no conhecimento e experiência dos despachantes. 
Para alocar viaturas às ocorrências, os despachantes devem atentar-se a várias restrições que as companhias elétricas devem respeitar como: o tempo que o cliente está sem fornecimento, a urgência da ocorrência, a distância da ocorrência às viaturas, o valor homem-hora $(\mathrm{HH})$ dos técnicos que atendem as ocorrências, horas excedentes caso o turno da viatura tenha se encerrado e quilometragem excedente em relação ao limite estipulado por veículo.

Em geral, a quantidade de despachantes é reduzida em relação à quantidade de ocorrências registradas. Além disso, não existe uma ferramenta ou sistema que auxilie especificamente na seleção da melhor viatura para atendimento de uma ocorrência, garantindo que o consumidor seja atendido no menor tempo possível e atendendo às restrições mencionadas.

O cenário descrito exibe alguns inconvenientes, tais como:

1. O conhecimento envolvido na tomada de decisão não é documentado. Apenas os despachantes humanos detêm tal conhecimento em suas mentes.

2. Não há ferramentas que possibilitem uma análise histórica e/ou estatística das decisões tomadas e das ocorrências registradas, não sendo possível obter otimização nas decisões.

3. Não se tem garantias da otimalidade das soluções concebidas.

4. Erros dispendiosos podem se repetir pois não é possível aprender com falhas de experiências anteriores [5].

Os inconvenientes apresentados revelam que companhias elétricas possuem um processo de tomada de decisão suscetível a falhas e não auditável. Além disso, o conhecimento e a memória organizacional não são documentados ou armazenados. Como o mecanismo de tomada de decisão não é um processo bem definido, o capital intelectual não é preservado.

Este artigo utiliza a CELG como escopo para elaborar um Sistema de Apoio à Decisão Inteligente (SADI) baseado em Sistemas Multi-Agentes (SMA) para auxiliar os despachantes de companhias elétricas nas tarefas de distribuição de viaturas e atendimento de falhas na rede elétrica.

As vantagens inicialmente observáveis são processamento distribuído de ocorrências, automatização, inteligência e sugestão de soluções com base em conhecimento armazenado (não apenas com base na experiência dos despachantes). 
Espera-se como consequência um aumento na velocidade de atendimento das ocorrências, minimização do tempo que o usuário fica sem fornecimento, redução dos custos com desperdício de recursos humanos e financeiros, e a preservação do capital intelectual e da memória organizacional das companhias elétricas, minimizando os problemas apresentados.

O restante deste trabalho está estruturado da seguinte forma: a Seção 2 discute os aspectos teóricos envolvidos na solução no problema; a Seção 3 apresenta o modelo proposto, discute a implementação e os conceitos utilizados; a Seção 4 apresenta os testes realizados; a Seção 5 traz as conclusões, trabalhos futuros e discussões finais sobre o tema. Por fim, são apresentadas as referências utilizadas neste trabalho.

\section{Fundamentação Teórica}

Um Sistema de Apoio à Decisão (SAD) é um Sistema de Informação (SI) que apoia qualquer processo de tomada de decisão em áreas de planejamento estratégico, controle gerencial e controle operacional. São sistemas que oferecem dados e modelos para solução de problemas [17].

Para que ele seja capaz de apoiar decisões é necessário que haja técnicas que capacitem o SAD a sugerir soluções. As técnicas de Inteligência Artificial (IA) têm sido disseminadas como modo de implementar a inteligência do SAD. Tais SADs são chamados de SADs Inteligentes (SADI) [18]. Dentre as técnicas de IA utilizadas para implementar a inteligência do SAD, agentes inteligentes tem sido uma das mais disseminadas [3].

Um agente é uma entidade lógica ou física que tem uma missão a ser executada de maneira autônoma e em coordenação com outros agentes. [12]. O agente é, em suma, uma unidade autônoma de resolução de problemas. [13]

Sistema Multi-Agente (SMA) refere-se à sub-área da Inteligência Artificial Distribuída que avalia o comportamento de um agrupamento de agentes que trabalham em conjunto para resolver um problema que está além das capacidades de um único agente [14].

Um SMA pode ser classificado em relação à natureza dos agentes que o compõe (heterogêneo, para um conjunto de agentes distintos; e homogêneo, para agentes idênticos) e em relação às capacidades cognitivas dos agentes que o compõe (reativos ou cognitivos). Os agentes podem ainda ser classificados em virtude de suas relações sociais (cooperativo, 
quando os agentes tem um objetivo comum e cooperam para atingi-lo; ou competitivo, quando os agentes competem para atingir seus objetivos ou consumir recursos) [15].

A arquitetura reativa implementa a tomada de decisão como um mecanismo de estímulo-resposta disparado por sensores. Agentes reativos não têm nenhum modelo simbólico central e não utilizam nenhum raciocínio complexo, ao contrário da arquitetura cognitiva [2].

Os SMA Reativos são caracterizados por utilizar agentes do tipo reativo. Não há planejamento de ações e a sociedade geralmente contém muitos agentes replicados. Exemplo: colméia de abelhas [15].

Apesar de agentes reativos não possuírem cognição, utilizá-los em um SADI não torna o sistema menos inteligente. Segundo Brooks, um comportamento inteligente pode ser gerado sem representação explícita e raciocínio abstrato providos pelas técnicas de Inteligência Artificial simbólica. Ele afirma ainda que a inteligência é uma propriedade emergente de sistemas complexos [16].

O SMA utilizado neste SAD é essencialmente reativo. Tal escolha não afetou a inteligência do sistema, endossando a afirmação de Brooks.

\section{Modelo Proposto}

Para resolver o problema descrito, propõe-se um modelo que sugere soluções para os despachantes com base em um processo bem definido. Tal processo tem como alicerce a troca de mensagens entre os agentes do sistema. O SMA utilizado é heterogêneo. Os agentes são do tipo reativo e possuem caráter cooperativo.

Iniciativas correlatas de utilizar agentes para implementar a inteligência do SAD foram encontradas na literatura. Em relação à utilização de agentes para auxiliar empresas de energia elétrica, Nagata e Sasaki [7] apresentam um trabalho no qual agentes auxiliam no processo de encontrar pontos de falha na rede elétrica de modo automático e notificar sobre a falha encontrada. Tolbert et al. [10] mostram um cenário no qual agentes auxiliam no gerenciamento, otimização do consumo e geração de energia. Rehtanz [9] menciona a utilização de agentes na operação e controle de sistemas de energia elétrica, auxiliando na gerência da rede, pontos de falha e fluxo de energia. Phadke e Thorp [8] sugerem a utilização de agentes para auxiliar no processo de retransmissão de energia. 
Não foi encontrado nenhum outro trabalho na literatura que utilizasse agentes para implementar a inteligência de um SADI para resolver problemas de distribuição de veículos para manutenção de redes de energia elétrica.

A Figura 1 apresenta a arquitetura em alto-nível do sistema. Em concordância com trabalhos presentes na literatura ([1], [4] e [11]), a arquitetura apresenta um agente especializado em recepcionar dados do usuário e comunicar aos agentes do SMA as requisições realizadas pelo usuário durante a interação com o SAD. Tal agente é chamado Agente Comunicador. Além do Agente Comunicador, existem ainda os Agentes Viatura, Despachante e Testes.

O modelo estabelece um processo para dar suporte ao trabalho desenvolvido em companhias elétricas, composto de duas fases principais: distribuição de viaturas em cada início de turno e alocação das viaturas às ocorrências (despacho) tomando as restrições mencionadas como parâmetro para escolha e/ou sugestão.

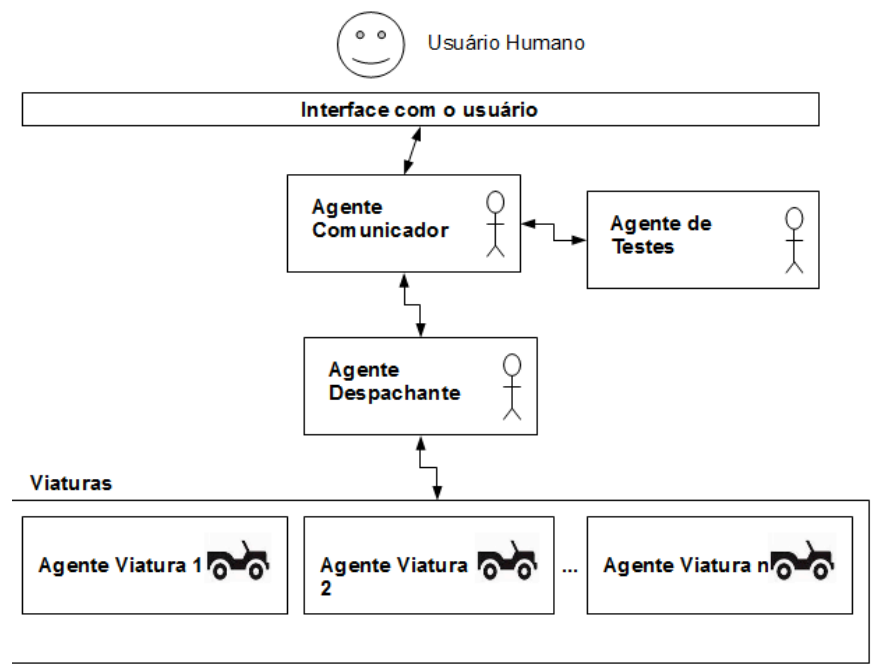

Figura 1. Arquitetura do sistema

A seção seguinte apresenta a solução proposta para a primeira fase do Processo de Atendimento de Ocorrências: a distribuição de viaturas nos inícios de turno.

\subsection{Distribuição de Viaturas}

Para a distribuição das viaturas na região de atendimento, utilizou-se uma base histórica de ocorrências da companhia. Esta base contém dados das ocorrências e das 
viaturas. Os dados das viaturas são: a posição geográfica e a quantidade de quilômetros rodados. Os dados das ocorrências são: o número identificador, a hora de requisição de atendimento do cliente, a hora da chegada da viatura, a hora do reestabelecimento do funcionamento da rede elétrica, a urgência, o número da prioridade, o tipo de problema informado pelo cliente e a posição geográfica.

Neste caso, o problema constitui-se na distribuição geográfica das viaturas, de acordo com o número de ocorrências, ou seja, alocar viaturas em regiões onde, historicamente, mais ocorrências foram registradas. As áreas de atuação das viaturas têm tamanho variável. Esta alocação inicial das viaturas (no início de cada turno de trabalho) em regiões próximas de onde se supõe que possam vir a ser registradas ocorrências contribuirá na redução do tempo de atendimento.

Poder-se-ia tentar identificar regiões mais propensas à falta de energia a partir da identificação de variáveis de risco, como por exemplo, a presença ou não de árvores nas ruas, idade da rede de distribuição, etc. Entretanto, esta previsão foge ao escopo deste trabalho.

O problema em questão poderia ser tratado com mais rigor de forma a buscar uma solução ótima, ou seja, encontrar a região (grupo de blocos de $1 \mathrm{~km}^{2}$ ) onde mais ocorrências tenham ocorrido no passado. Poder-se-ia também identificar a quantidade ideal de blocos (a extensão) em cada região. Deve-se observar que as áreas das regiões não são as mesmas, uma vez que a concentração de consumidores é variável nas diversas regiões das cidades.

Como uma distribuição ótima dos recursos (viaturas), em função de ocorrências passadas, não garante uma melhoria no tempo de atendimento para ocorrências futuras, optou-se por não buscar esta distribuição ideal, mas sim uma solução aproximada que pudesse trazer ganhos com relação à situação atual. Vale à pena ressaltar que, no caso da CELG, esta distribuição é feita apenas duas vezes ao ano, segundo critérios tácitos do gerente do setor.

Em vista do exposto, uma heurística, chamada de Heurística da Diagonal, foi criada para resolver o problema da distribuição de viaturas a cada início de turno. Esta heurística processa os dados históricos de ocorrências na região de atendimento para distribuir as viaturas de modo proporcional à quantidade de ocorrências registradas em cada porção da área de atendimento.

Para a execução da heurística, seleciona-se um período (por exemplo, um mês) no histórico de ocorrências. Cada ocorrência possui um registro de posicionamento geográfico para que se possa identificar onde ela ocorreu dentro da área de atendimento. A quantidade de viaturas é fixa para todos os turnos (no caso desta instância do problema, 154 viaturas). Divide-se então a quantidade total de ocorrências pela quantidade total de viaturas obtendose um valor que representa a média de ocorrências atendidas por viatura (moav) no período 
considerado. Constrói-se uma matriz que representa a incidência de ocorrências na região de atendimento.

Para construir a matriz de incidência de ocorrências, divide-se a área de atendimento em blocos de $1 \mathrm{~km}^{2}$. Identifica-se então quantas ocorrências foram registradas em cada bloco no período. Em seguida, cria-se agrupamentos de blocos para a distribuição de viaturas, varrendo-se a matriz a partir do canto superior esquerdo e crescendo-se diagonalmente (por isso o nome "Heurística da Diagonal"). O agrupamento começa na linha 1 e coluna 1, e se necessário, cresce na diagonal para a linha 2 e coluna 2, formando um grupo com as células $(1,1),(1,2),(2,1)$ e $(2,2)$. Este processo irá se repetir até que a somatória das ocorrências seja igual ou superior ao moav ou tenha chegado em alguma extremidade ou não seja possível expandir por causa de um outro agrupamento a direita.

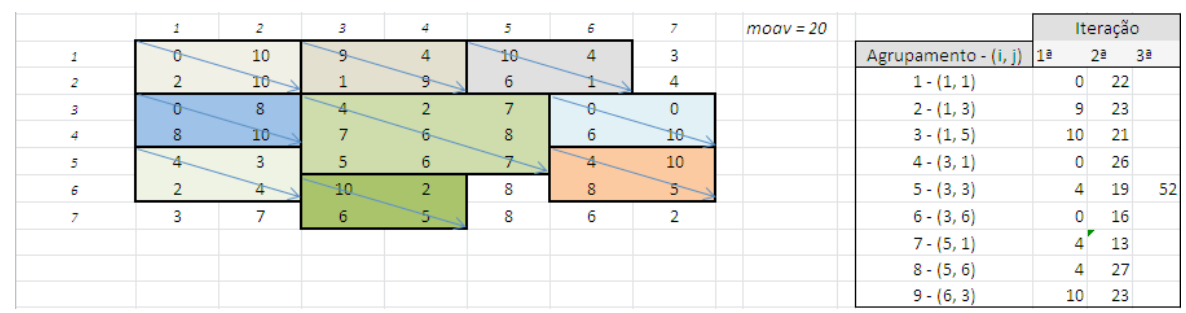

Figura 2. Exemplo Execução da Heurística da Diagonal sobre região de atendimento mapeada com Problema das Fronteiras destacado.

A Figura 2 representa uma área de atendimento. Cada célula da tabela representa um bloco geográfico de $1 \mathrm{~km}^{2}$ e os números em cada célula representam a quantidade de ocorrências registradas naquele bloco durante o período considerado. Na primeira iteração, para criar o primeiro agrupamento, verifica-se que a quantidade de ocorrências no bloco $(1,1)$ é inferior ao moav, não sendo viável alocar uma viatura àquela região. Então, executa-se a segunda iteração, expandindo-se a área analisada no sentido da diagonal. Com isso a somatória das ocorrências registradas na região selecionada (de $(1,1)$ a $(2,2))$ no histórico é maior que o moav, o que torna viável alocar àquela região uma viatura para atendimento.

O processo descrito repete-se ao longo da primeira linha até que não seja mais possível criar agrupamentos como os visíveis na Figura 2. Esta situação ocorre quando não restam blocos suficientes para atingir o valor do moav e que impossibilitam expandir na diagonal. Tal fenômeno será tratado na seção seguinte.

Quando a linha acaba, o processo inicia-se novamente na próxima linha seguinte que permita formação de novo agrupamento, como no caso do agrupamento que inicia na célula $(3,1)$ da Figura 2. Este processo continua até que todos os grupos sejam formados e não seja mais possível criar novos blocos através da expansão diagonal. 


\subsection{Problema das Fronteiras}

Inicialmente, a Heurística da Diagonal era considerada satisfatória para um bom resultado. Entretanto, foi identificado um problema intitulado de "Problema das Fronteiras". Este problema acontece quando, após a criação dos agrupamentos, seguindo uma sequência de uma linha, restam células desagrupadas na vertical ou horizontal.

Para evitar tal fenômeno, como aconteceu na Figura 2, convencionou-se que, um agrupamento será expandido se houver células à direita formando uma linha vertical, como nos agrupamentos três e nove da Figura 2, ou se houver células abaixo formando uma linha horizontal, como nos agrupamento sete e oito da Figura 3.

A Figura 3 mostra um exemplo no qual o Problema das Fronteiras foi solucionado. Depois que a Heurística da Diagonal é executada, a região de atendimento fica dividida em agrupamentos. Uma viatura será alocada para cada agrupamento, para atender às ocorrências daquela região. Caso restem viaturas após esta distribuição inicial, estas serão rateadas proporcionalmente ao número de ocorrências entre os agrupamentos.

\begin{tabular}{|c|c|c|c|c|c|c|c|c|c|c|c|c|}
\hline & 1 & 2 & 3 & 4 & 5 & 6 & 7 & moav $=20$ & & \multicolumn{3}{|c|}{ Iteração } \\
\hline 1 & 0 & 10 & 9 & 4 & 10 & 4 & 3 & & Agrupamento - $(\mathrm{i}, \mathrm{j})$ & $1 \mathrm{a}$ & 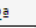 & $3 \mathrm{a}$ \\
\hline 2 & 2 & 10 & 1 & 9 & 6 & 1 & 4 & & $1-(1,1)$ & 0 & 22 & \\
\hline 3 & 0 & 8 & 4 & 2 & 7 & 0 & 0 & & $2-(1,3)$ & 9 & 23 & \\
\hline 4 & 8 & 10 & 7 & 6 & 8 & 6 & 10 & & $3-(1,5)$ & 10 & 28 & \\
\hline 5 & 4 & 3 & 5 & 6 & 7 & 4 & 10 & & $4-(3,1)$ & 0 & 26 & \\
\hline 6 & 2 & 4 & 10 & 2 & 8 & 8 & 5 & & $5-(3,3)$ & 4 & 19 & 52 \\
\hline 7 & 3 & 7 & 6 & 5 & 8 & 6 & 2 & & $6-(3,6)$ & 0 & 16 & \\
\hline & & & & & & & & & $7-(5,1)$ & 4 & 23 & \\
\hline & & & & & & & & & $8-(5,6)$ & 4 & 35 & \\
\hline & & & & & & & & & $9-(6,3)$ & 10 & 39 & \\
\hline
\end{tabular}

Figura 3. Solução do Problema das Fronteiras

\subsection{Atendimento das Ocorrências}

Para cada ocorrência registrada, o Agente Despachante trocará mensagens com os Agentes Viatura para deliberar sobre qual a viatura mais adequada para atender a ocorrência, observando às restrições de distância, turno, custo homem-hora e urgência.

A Figura 4 ilustra todo o processo de obtenção de uma sugestão de viaturas para atender a uma ocorrência, desde a requisição do usuário até a sugestão das viaturas mais adequadas. Os passos seguidos são: registrada.

1. O despachante humano requisita sugestão de viaturas para uma ocorrência 


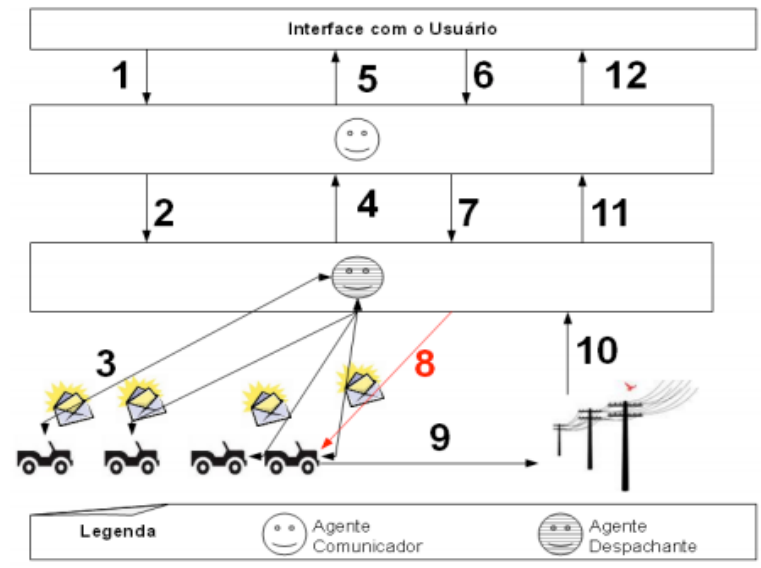

Figura 4. Processo Completo de Sugestão de Viaturas.

2. Agente Comunicador encaminha requisição de sugestão de conjunto de viaturas para atender a uma ocorrência ao Agente Despachante.

3. O Agente Despachante troca mensagens com os Agentes Viatura e requisita o cálculo da distância entre as viaturas e a ocorrência considerando as posições das viaturas e da ocorrência (valor passado na mensagem).

4. O Agente Despachante retorna a lista das viaturas mais próximas e disponíveis mediante a urgência da ocorrência.

5. O Agente Comunicador dispõe as viaturas sugeridas pelo Agente Despachante.

6. O despachante humano escolhe uma viatura.

7. O Agente Comunicador notifica o Agente Despachante sobre a viatura escolhida pelo usuário.

8. O Agente Despachante notifica o Agente Viatura escolhido para que ele desloque-se até o local da ocorrência.

9. A viatura muda seu status para ocupada e desloca-se para reparar a falha na rede.

10. Após reparar a falha na rede, o Agente Viatura notifica o Agente Despachante da conclusão do seu trabalho. 
11. O Agente Despachante recebe a notificação de término e a encaminha para o Agente Comunicador.

12. O Agente Comunicador notifica o despachante humano do término, o que encerra aquele ciclo de atendimento.

A troca de mensagens entre Agentes Viatura e Despachante dispara o cálculo de distância nos Agentes Viatura e o despacho no Agente Despachante.

O SMA provê a capacidade de representação de várias viaturas trabalhando de forma paralela nas diversas sub-regiões da área de atendimento. Recursos como a troca de mensagens e controle autônomo da sincronização dos agentes foram alguns dos critérios utilizados para aplicar a tecnologia de agentes à solução do problema. Outras tecnologias como Threads e Objetos Distribuídos forçam o programador a controlar o paralelismo e a troca de mensagens é baseada em chamada de métodos ao invés do conceito de caixa de mensagens como é o caso dos agentes.

\section{Testes}

Um protótipo foi desenvolvido utilizando o arcabouço JADE [6]. Para aferir a eficiência do SAD foram conduzidos testes utilizando um histórico de tratamento de ocorrências. Este histórico foi cedido pela CELG e ilustra uma amostra de dados coletados referentes ao atendimento de ocorrências no mês de Agosto de 2009. Destes dados, o dia 29/08/2009 foi selecionado para os testes por ser o dia com maior número de ocorrências.

Para cada viatura que trabalhou no dia, foram analisados a Identificação da viatura, Horário de início de turno, Horário de fim de turno, a Localização (posição geográfica) inicial, Distância percorrida por cada viatura no histórico. Para cada ocorrência, foram analisadas a Identificação da ocorrência, Hora da criação da ocorrência, Hora do início do atendimento, Tempo de atendimento da ocorrência no histórico, a urgência do atendimento, Prioridade de atendimento, Tipo de serviço, Localização (posição geográfica), Viatura associada (a que atendeu originalmente a ocorrência), Distância percorrida pela viatura que atendeu originalmente a ocorrência.

Os testes foram conduzidos simulando o despacho automatizado das viaturas para atendimento das ocorrências. Para este despacho convencionou-se que sempre seria usada a viatura que fosse a melhor sugestão fornecida pelo SAD. O objetivo principal desta política é reduzir o tempo de espera que uma ocorrência fica aguardando até ser atendida. Para conduzir os testes foi criado um Agente de Testes. Tal agente seleciona as viaturas de acordo com a política estabelecida.

A simulação foi conduzida durante 16 horas (dois turnos), criando-se no sistema as ocorrências na mesma posição geográfica em que elas estavam registradas na base histórica. 
Foram criadas 267 ocorrências e 48 viaturas (a mesma quantidade dos dados da CELG). Entretanto, as viaturas foram posicionadas de um modo diferente em relação ao posicionamento feito pelos despachantes humanos no cenário real. A intenção era mostrar que o posicionamento sugerido pelo sistema é melhor do que o realizado costumeiramente de maneira ad-hoc e baseado na experiência dos despachantes humanos. Ao longo de 16 horas as ocorrências foram atendidas pelas viaturas sugeridas pelo sistema, produzindo dados que permitissem a comparação do desempenho dos despachantes humanos sozinhos com o desempenho do SAD na tarefa de atendimento de ocorrências em condições semelhantes.

Os dados aferidos como resultado da simulação foram a quilometragem total percorrida pelas viaturas, o tempo de espera para uma ocorrência ser atendida (quanto tempo uma ocorrência demorou para que fosse determinada uma viatura para atendê-la), média de tempo de espera total (determina quanto tempo decorreu, em média, para que uma ocorrência fosse resolvida por completo - alocação de viatura, deslocamento até o ponto da falha e tempo de reparo) e desvio padrão da quilometragem das viaturas (já que existe uma diferença muito grande entre as quilometragens percorridas por cada viatura em um dia). A velocidade média das viaturas utilizada para a simulação foi de $40 \mathrm{Km} /$ Hora.

A Tabela 1 mostra uma comparação dos resultados obtidos utilizando o SAD e um histórico de atendimento de ocorrências durante um período de 16 horas, no qual despachantes humanos efetuaram escolhas sem auxílio do SAD.

O histórico de dados reais mostra que a média do tempo de espera para iniciar o atendimento de uma ocorrência era de aproximadamente 28 minutos, enquanto que o SAD é capaz de deliberar sobre a melhor viatura para atender a uma ocorrência em 3 segundos, em média.

Tabela 1. Resultados dos Testes

\begin{tabular}{|l|c|c|c|}
\hline & Dados Históricos & Simulação & Melhoria \\
\hline Quilometragem total percorrida (Km) & 1801,39 & 1348,51 & 452,88 \\
\hline Média de Tempo de Espera & $00: 28: 01$ & $00: 00: 03$ & $00: 27: 58$ \\
\hline Desvio Padrão (Km) das viaturas & 25,73 & 16,75 & 8,98 \\
\hline Média de Espera Total & $00: 45: 56$ & $00: 26: 47$ & $00: 19: 09$ \\
\hline
\end{tabular}

A quilometragem percorrida pelas viaturas no histórico foi de pouco mais que 1800 quilômetros enquanto que na, simulação, a quilometragem percorrida foi de pouco menos que 1350 quilômetros. Isso mostra que as viaturas trafegam menos porque há uma escolha 
mais criteriosa a respeito de qual está mais próxima da ocorrência e disponível para atendimento.

A média de tempo de espera para a conclusão de um atendimento foi reduzida quase pela metade e a variação de quilometragem percorrida pelas viaturas foi minimizada, o que mostra uma distribuição mais igualitária da carga de trabalho sobre as viaturas.

Os resultados mostram que o SAD proporcionaria, se implantado na CELG, uma economia média de 452,88 quilômetros em dois turnos (226,44 quilômetros por turno), reduziria o tempo de acionamento de um atendimento, e reduziria o desvio padrão da quilometragem das viaturas, promovendo um trabalho mais equilibrado a todas as viaturas, reduzindo sobrecarga de trabalho sobre algumas das viaturas, e consequentemente reduzindo gastos com horas extras e outros custos.

Portanto, pode-se afirmar que o SAD é efetivo em sua tarefa de minimizar os custos e prazos dos atendimentos, proporcionando benefícios relevantes para companhias elétricas e atingindo os objetivos propostos no início deste trabalho.

\section{Conclusões e Trabalhos Futuros}

Conclui-se, portanto, que a utilização de Sistemas Multi-Agentes para a implementação de Sistemas de Apoio à Decisão Inteligentes é uma alternativa viável para minimizar custos e otimizar o Processo de Atendimento de Ocorrências em companhias elétricas. Além disso, os resultados mostram que a tecnologia de agentes é adequada não apenas para o cenário descrito, mas para a implementação da inteligência de SAD de modo genérico.

Este tipo de abordagem protege o capital intelectual da corporação e a memória organizacional. As decisões, antes tomadas com base exclusivamente na experiência dos despachantes humanos, tornam-se agora uma cooperação entre a sugestão oferecida pelo sistema - regido por um processo definido - e a decisão do despachante humano. como:

Este artigo possui propostas que podem ser estendidas em trabalhos futuros, tais

1. Heurística da Diagonal: que pode ser investigada para aplicação em outras áreas de conhecimento que requeiram uma concentração de elementos em um determinado espaço.

2. A própria proposta de utilização de Agentes para a Inteligência do SAD aplicada aos Problemas do Setor Elétrico pode ser estendida para aumentar a inteligência dos Agentes Viatura, convertendo-os em agentes cognitivos, capazes de deliberar por si próprios e negociar com os agentes de seu agrupamento qual a melhor opção para atender às ocorrências que chegam. Pode-se também aumentar a quantidade de agentes do tipo despachante de modo a dar ao modelo um caráter mais distribuído, criando um Agente 
Despachante para cada agrupamento de blocos, minimizando a sobrecarga de mensagens trocadas entre os Agentes Viatura e Despachante. Com isso, cria-se no SAD conceitos como a negociação de viaturas entre Agentes Despachantes, que deliberariam e "emprestariam" viaturas sob sua responsabilidade.

O modelo proposto é efetivo para auxiliar os despachantes de qualquer companhia elétrica na seleção das viaturas mais adequadas para atender as ocorrências, com o menor tempo de espera por parte do consumidor e menor custo associado a recursos humanos e financeiros. Espera-se que com a adoção deste modelo como solução em companhias elétricas, surja uma nova forma de realizar a alocação de viaturas e executar tarefas de manutenção das redes elétricas: uma forma mais inteligente, precisa e que preserve o conhecimento adquirido ao longo dos anos nestas organizações.

\section{Agradecimentos}

À CELG pelo fornecimento dos dados que fomentaram os testes realizados.

\section{Referências}

[1] Addis A., Armano G. e Vargiu E. From a Generic Multi-Agent Architecture to MultiAgent Information Retrieval Systems. Proceedings of Sixth International Workshop From Agent Theory to Agent Implementation. pp. 3-9. 2008.

[2] Bellifemine F. L., Caire G. e Greenwood D. Developing Multi-Agent Systems with JADE. Wiley, Inglaterra, 2007.

[3] Eom, S E; Kim, E. A Survey of Decision Support System Applications (1995-2001). Journal of the Operational Research Society, pp. 1264-1278, vo. 57, no. 11, 2006.

[4] Czibula G., Guran A. M. e Czibula, G. S. C. I. G. Multi-Agent Decision Support Systems Based on Supervised Learning. Proceedings of IEEE International Conference on Automation, Quality and Testing, Robotics - Volume 03, p. 353-358. 2008.

[5] Vasconcelos, J. B. de, Rocha, A., e Kimble, C. Organisational Memory Information Systems: An Example of a Group Memory System for the Management of Group Competencies. Journal of Universal Computer Science - Volume 9, pág. 1410-1427, no. 12, 2003.

[6] Telecom Italia. JADE - Java Agent Development Framework. Disponível em: http://jade.tilab.com/. Acesso em: 24 jan. 2009, 2009. 
[7] T. Nagata and H. Sasaki. A Multi-Agent Approach to Power System Restoration. IEEE Transactions on Power Systems, IEEE Press. Volume 17, p 457-462, número 2, 2002.

[8] Phadke, A. e Thorp, J. Computer Relaying for Power Systems. Wiley, Inglaterra 2009.

[9] Rehtanz, C. Autonomous Systems and Intelligent Agents in Power System Control and Operation. Springer-Verlag, EUA, 2003.

[10] Tolbert, L. M., Qi, H, e Peng, F. Z. Scalable Multi-Agent System for Real-Time Electric Power Management. IEEE Power Engineering Society Summer Meeting. IEEE Power Electronics Society. p. 1676-1679, 2001.

[11] Yu T., Zhou, J., Xu, F., Gong, Y. e Wang, W. Decision support system of product development based on multi-agent. Proceedings of International Conference on Information Technology and Computer Science. pp. 305-308. 2009.

[12] Hübner, J. F.; Sichman, J S. Aplicação de Organização de Sistemas Multi-Agentes em Futebol de Robôs. Anais da XI Escola de Informática da Sociedade Brasileira de Computação, p. 1-19. 2003.

[13] Wooldridge, M; Jennings, N. R. Intelligent Agents: Theory and Practice. The Knowledge Engineering Review. Cambridge University Press. Volume 10, p 115-152, número 2, 1995.

[14] O’Hare, G M P, Jennings, N. R. Foundations of Distributed Artificial Intelligence. John Wiley \& Sons. Canadá, 1996.

[15] Russell, S J; Norvig, P. Artificial Intelligence - A Modern Approach, Prentice Hall, Estados Unidos, 2010.

[16] Brooks, R A. Intelligence without Representation. Artificial Intelligence. Elsevier Science Publishers, Volume 47, p 139-159, número 1, 1991.

[17] Sprague, R H, Hugh J W. Sistemas de Apoio à Decisão, Campus, Brasil,1991.

[18] Arnott, D; Pervan G. Eight Key Issues for the Decision Support Systems Discipline. Decision Support Systems. Elsevier Science Publishers B V. Volume 44, p 657-672, Issue 3, 2008. 